\title{
Electrophoretic Carbon Nanotube Field Emission Layer for Plasma Display Panels
}

Qifa Liu, Zhuoqing Yang, Yan Wang, Guifu Ding*

(Received 15 November 2012; accepted 26 November 2012; published online 6 December 2012)

\begin{abstract}
A carbon-nanotube (CNT) electrophoretic deposition (EPD) process has been developed to prepare a field emission layer in plasma display panels (PDP) for discharge voltage reduction. The CNT layer as a source of discharge priming electrons has been fabricated on the PDP front panel. The balling grinding, mix-acid treatment and EPD parameters have been investigated in order to obtain good uniformity and excellent field emission capability of CNT layer, in order to meet the specifications of CNTs in PDP cell. The measured turn-on field was around $1.1 \mathrm{~V} / \mu \mathrm{m}$ in the field emission testing while the minimum sustaining voltage was decreased by $30 \sim 40 \mathrm{~V}$ with the use of CNT layer in the discharge testing.
\end{abstract}

Keywords: EPD; Field emission; CNT; PDP; Sustaining voltage

Citation: Qifa Liu, Zhuoqing Yang, Yan Wang and Guifu Ding, "Electrophoretic Carbon Nanotube Field Emission Layer for Plasma Display Panels", Nano-Micro Lett. 4 (4), 247-252 (2012). http://dx.doi.org/10. $3786 / \mathrm{nml}$.v4i4.p247-252

\section{Introduction}

High operating voltage is still one of the major issues to overcome in current plasma display panels (PDP), especially in large-sized panel market. There have been several attempts to reduce the PDP operating voltage: Adding auxiliary structure on coplanar electrode [1] or front dielectric surface [2]; inducing a partial strong electric field in the cell space, resulting in the decrease of the minimum sustain voltage. Enhancing secondary electron emission capability of the protective layer is another effective way to significantly decrease the discharge voltage. Alternatively, PDP operating voltage reduction can be achieved by changing the $\mathrm{MgO}$ surface morphology and crystal structure by annealing process [3] or via altering preparation conditions [4] to increase the secondary electron emission. Last but not least, PDP operating voltage reduction can be achieved by developing the composite protective layer, for example, $\mathrm{SrO} / \mathrm{MgO}[5], \mathrm{MgO} /$ Graphite-in-diamond (GiD) [6], $\mathrm{MgO} / \mathrm{TiO}_{2}$ [7], $\mathrm{MgO} /$ nano $\mathrm{Au}$ [8], $\mathrm{ZnO} / \mathrm{MgO}$ [9] and $\mathrm{MgO} / \mathrm{NiO}$ [10], in order to increase the secondary electron emission. The driving voltage could be reduced in the range of $10 \sim 100 \mathrm{~V}$ by the above methods.

A special electrophoretic deposition (EPD) technique for carbon-nanotube (CNT) auxiliary layer preparation has been developed in this investigation, playing a role as the source of field-emission electrons in PDP cell while the lower discharge voltage was realized by the increasing priming electrons. Good uniformity and excellent field emission capability of CNT layer are the important factors enhancing its application in PDP cells. EPD is suitable for preparing this kind of CNT layers by using special technical processes and controlling process parameters. Electrophoretic CNT layer has been prepared at specified area on the dielectric layer of the front panel. This kind of field emission layer has the same function as the above examples, i.e., reducing the discharge voltage by increasing the electrons in PDP cell.

\section{Experimental}

The front panel of PDP with CNT field emission layer

National Key Laboratory of Science and Technology on Nano/Micro Fabrication Technology, Research Institute of Micro/Nano Science and Technology Shanghai Jiao Tong University, Shanghai 200240, China

*Corresponding author. E-mail: gfding@ sjtu.edu.cn 


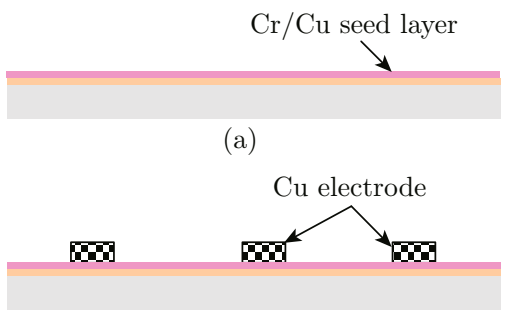

(b)

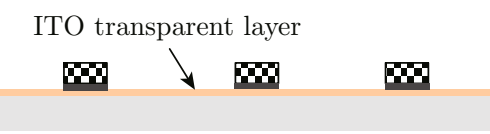

(c)

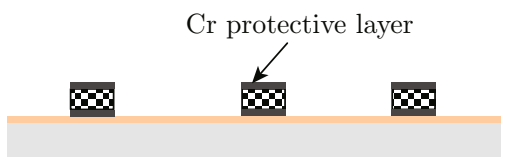

(d)

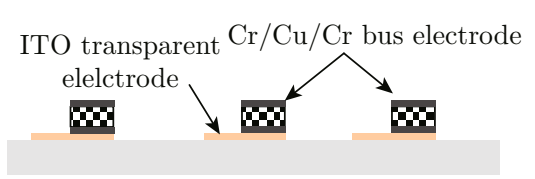

(e)

Glass dielectric layer

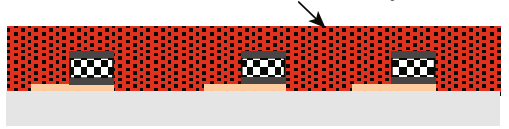

(f)

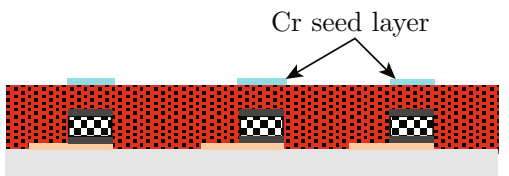

(g)

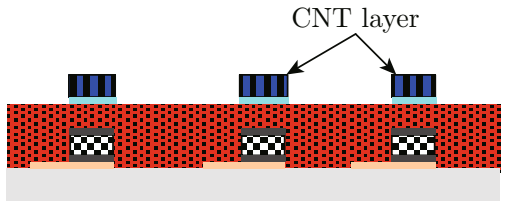

(h)

Fig. 1 Microfabrication processes of PDP front panel with CNT auxiliary layers.

was fabricated by surface-micromachining technology. The main fabrication process steps are described and sketched in Fig. 1(a)-(h) as follows.

a. Chromium/Copper $(\mathrm{Cr} / \mathrm{Cu})$ was sputtered as the seed layer on an ITO glass substrate for the electroplating of $\mathrm{Cu}$ electrode.

b. Photoresist spin coating and lithography were performed and then $\mathrm{Cu}(3-5 \mu \mathrm{m})$ was electroplated on the $\mathrm{Cr} / \mathrm{Cu}$ seed layer.

c. Remove the photoresist and the seed conducting layer. Photoresist spin coating and lithography were performed.

d. Sputter $\mathrm{Cr}(0.1 \mu \mathrm{m})$ on $\mathrm{Cu}$ electrode strip and photoresist. Make a Lift-off of $\mathrm{Cr}$ layer to form the $\mathrm{Cr} / \mathrm{Cu} / \mathrm{Cr}$ bus electrode.

e. Photoresist spin coating and lithography were performed to develop the area not needing ITO. ITO was etched to form the transparent electrode. Remove the photoresist.

f. Glass dielectric layer was fabricated by screen printing and sinter.

g. Photoresist spin coating and lithography were performed. Sputter Cr seed layer.

h. Lift-off of Cr layer to leave the seed layer where CNT layer will be fabricated. The CNT film was deposited on seed conducting layer by EPD. Initially, balling grinding was used to CNTs with tube diameters ranging from 20 to $30 \mathrm{~nm}$, and then boiled in mixed acids $\left(\mathrm{H}_{2} \mathrm{SO}_{4}: \mathrm{HNO}_{3}=3: 1\right)$ at $60^{\circ} \mathrm{C}$ for $2 \sim 4 \mathrm{~h}$. Then, CNTs in given concentration were dispersed in distilled water and $0.5 \sim 2 \mathrm{~g} / 1 \mathrm{Mg}\left(\mathrm{NO}_{3}\right)_{2} \cdot 6 \mathrm{H}_{2} \mathrm{O}$ as the charger salt was added into the suspension. And then the sus- pension was sonicated for 1-2 h at room temperature to produce a homogeneous suspension. The CNT film was deposited on the conducting layer by EPD technique. The composition and the process conditions of EPD are listed in Table 1. After the EPD process, the substrate was carefully pulled upwards from the EPD cell to avoid any influence of drag force between the suspension and the surface of the deposited film. The panel was dried slowly in air at room temperature and in horizontal position to achieve a homogeneous and smooth surface of the CNT films.

Table 1 The composition content and operation conditions of electrophoretic deposition

\begin{tabular}{ll}
\hline Items & Parameters \\
\hline Solvents & $\mathrm{H}_{2} \mathrm{O}$ \\
$\mathrm{CNTs}$ & $0.2 \sim 0.7 \mathrm{~g} / 1$ \\
$\mathrm{Mg}\left(\mathrm{NO}_{3}\right)_{2} 6 \mathrm{H}_{2} \mathrm{O}$ & $0.5 \sim 2 \mathrm{~g} / \mathrm{l}$ \\
$\mathrm{pH}$ & $4 \sim 6$ \\
Temperature & $20 \sim 30^{\circ} \mathrm{C}$ \\
DC electric field & $10 \sim 30 \mathrm{~V} / \mathrm{cm}$ \\
Electrode distance & $1 \sim 3 \mathrm{~cm}$ \\
\hline
\end{tabular}

Electron field-emission testing and discharge testing was carried out by the setup shown in Fig. 2. Field-emission property was measured at a pressure of $1.5 \times 10^{-5} \mathrm{~Pa}$. The electric field is the support DC voltage divided by distance between two CNT stripe layers. For discharge testing, a Ne-Xe gas mixture with the total pressure 450 Torr was used as the discharge gas, and the Xe content was fixed at $7 \%$. The frequency of the driving voltage waveform for sustain discharges was 50 
$\mathrm{kHz}$, and the width was $10 \mu \mathrm{s}$. Rear panel was just to form the cell space with front panel.

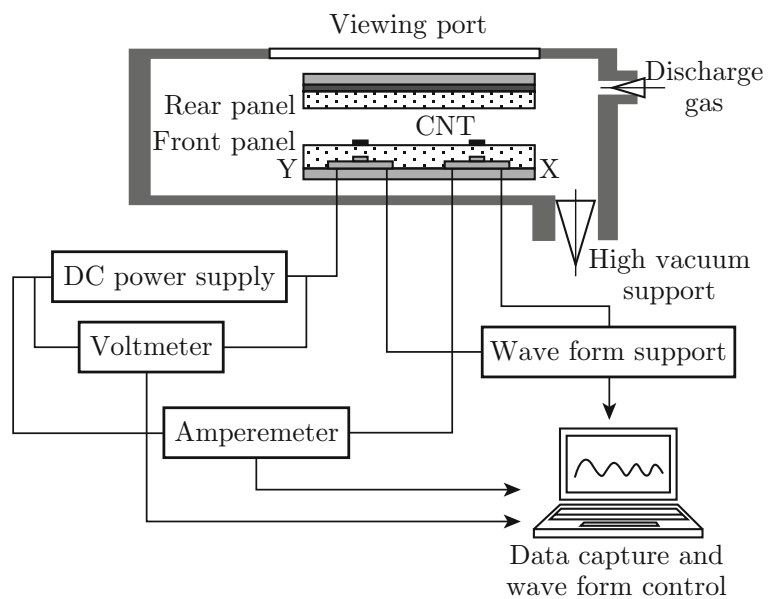

Fig. 2 Sketch of the test setup for CNT field emission testing and PDP discharge testing.

\section{Results and discussion}

EPD is achieved via the motion of charged CNTs, dispersed in a suitable solvent, towards an electrode under an applied electric field. EPD offers advantages of low cost, process simplicity, uniformity of deposits, controllable thickness, microstructural homogeneity, and deposition on complex shaped substrates [11, 12]. The pretreatment to $\mathrm{CNT}$ is to obtain pure and dispersed emitting materials. As is well known, CNT has excellent field emission characteristics and possesses the advantages of high field enhancement factor, low threshold field, chemical inertness and thermal stability. CNT may be the right material for producing priming electrons in PDP. However, as-grown carbon nanotubes are intrinsically inert, often aggregated or entangled, and may contain impurities (such as amorphous carbon or catalytic metal particles).

Balling grinding was used for good dispersion and defects increase. A mixture of concentrated nitric and sulphuric acids can simultaneously purify, shorten and functionalize CNTs. Under such aggressive conditions, defective sites in the CNTs are attacked, resulting in the formation of fragmented CNTs decorated with carboxylic acid and other oxygen-containing groups on their surface. Figure 3(a) shows the FT-IR spectra of CNTs before and after the above pretreatment, which proved these groups functionalization of CNTs. 1080 $\mathrm{cm}^{-1}$ is $\mathrm{O}-\mathrm{H}$ deformation vibrations and $1166 \mathrm{~cm}^{-1}$ is assigned to $\mathrm{C}-\mathrm{O}$ stretching vibrations; $1378 \mathrm{~cm}^{-1}$ is $\mathrm{C}-\mathrm{O}$ single bond stretching and $1400 \mathrm{~cm}^{-1}$ is $\mathrm{O}-\mathrm{H}$ bending; $1650-1750 \mathrm{~cm}^{-1}$ is $-\mathrm{C}=\mathrm{O}$ stretching vibrations. These acidic groups electrostatically stabilize the CNTs in water, or other polar liquids. The Raman spectra curves of as-grown CNTs and CNTs after pretreatment are shown in Fig. 3(b). The D band and $\mathrm{G}$ band in two curves locate at the approximately same wavenumber of $1340 \mathrm{~cm}^{-1}$ and $1570 \mathrm{~cm}^{-1}$ in the spectra respectively. It is well known that Raman $\mathrm{G}$ band implies electronic property while D band shows information on defects. CNTs have higher peaks at both $\mathrm{D}$ band and $\mathrm{G}$ band after pretreatment, which illustrates purity improvement and defects increase. Meanwhile, we can also deduced from the Raman spectra that the relative peak intensities $I_{D} / I_{G}$ becomes bigger after pretreatment, which further implies the increased defects. Figure 4 shows the Gaussian grain diameter distribution of CNTs in water before and after the pretreatment. Compared with Fig. 4(a), the decrease of grain diameter and more regular normal distribution of Fig. 4(b), illustrates effective improvement on conglomeration and dispersibility of CNTs.
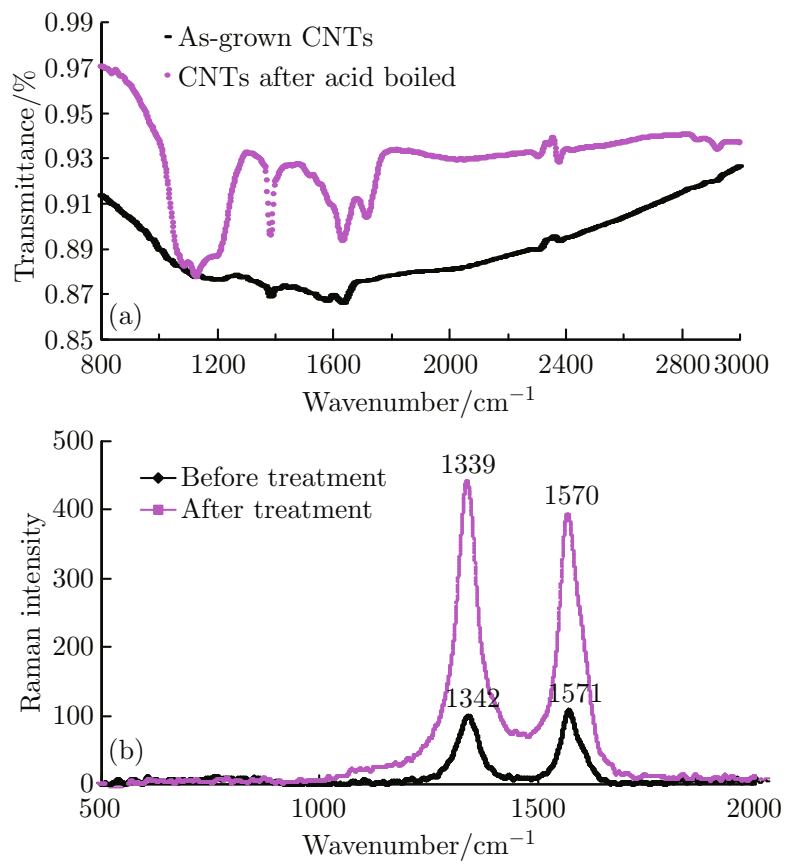

Fig. 3 FT-IR spectra (a) and Raman spectra (b) of CNT.

Besides the pretreatment, to satisfy the demand of CNT layer used in PDP cell, EPD operation parameters were also important factors. Low electric field strength results in poor quality, low density, non-uniform coatings, which do not adhere to the substrate. High electric field is necessary to deposit uniform and homogeneous CNT films on metallic substrates. However, higher electric field strengths or longer deposition times CNT aggregates are deposited rather than individual nanotubes, resulting in a large scatter of the yield and in poorer homogeneity of the CNT films [13, 14]. $\mathrm{Mg}\left(\mathrm{NO}_{3}\right)_{2} \cdot 6 \mathrm{H}_{2} \mathrm{O}$ as the charger salt was added into the suspension. It has been shown that the presence of charger salts can play an important role in improving the adhesion of CNTs to substrates and in increasing 


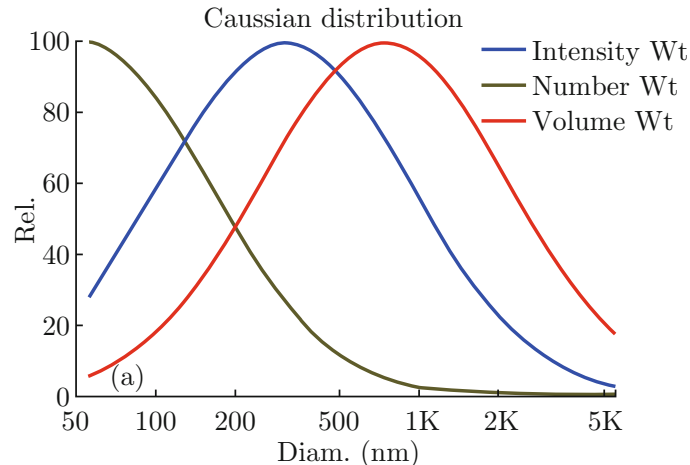

Caussian distribution

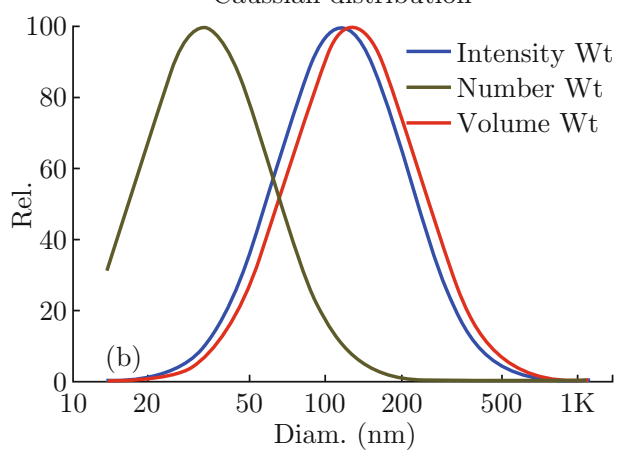

Fig. 4 Gaussian grain diameter distribution map of asgrown (a) and after pretreatment (b) CNTs dispersed in water.

the deposition rate in the EPD process [15-18]. In order to minimize the effect on PDP transmittance, the CNT layer is aligned above the bus electrode. Hence EPD layer with dense CNTs could be deposited on the specified area with strong electric field of PDP panel. Figure 5 presents the SEM picture and 3-dimensional micrograph of EPD CNT layer utilizing CNT before and after pretreatment. Homogeneity of the microstructure, uni- form coating thickness and sufficient adherence to the substrate are the character of EPD layer with CNTs after effective pretreatment. We observed coarse surface morphology and irregular CNTs distribution of EPD layer with no treatment CNTs, resulting from the entanglement and poor dispersion of CNTs in the solvent.

Figure 6 shows the plot of the field-emission current density versus the applied electric field. The current density of CNTs emitter increases with the applied field. The measured turn-on field to extract a current density of $10 \mu \mathrm{A} / \mathrm{cm}^{2}$ is around $0.8 \mathrm{~V} / \mu \mathrm{m}$. It is reasonable that the improved field electron emission of EPD CNTs is due to their pure CNTs with uniform and dense morphology. And CNTs after pretreatment provides good electron emission performances for CNT auxiliary layers. The low turn-on field of CNT auxiliary layer plays a beneficial role for PDP discharge. The well-worked PDP cell has strong electric field and it maybe distorted during the plasma formation. The distortion of the electric field is favorable for CNT field emission which induces more electrons released. And in the design, field emission layers was arranged within both cathode and anode region. Consequently, emitted electrons produced alternately from auxiliary layer in every discharge period, makes the CNTs layer work more efficiently. Figure 7 shows the discharge photos. The PDP panel with CNT layer lightened at $180 \mathrm{~V}$, while the traditional-type panel lightened at $220 \mathrm{~V}$. Because the influence of CNT layer, however, the discharge uniformity seems not as good as that of no CNT layer ones. Further research needs to be carried out to minimize this disadvantage. For example, improve the uniformity, thickness, area and consistency of the CNT layer.
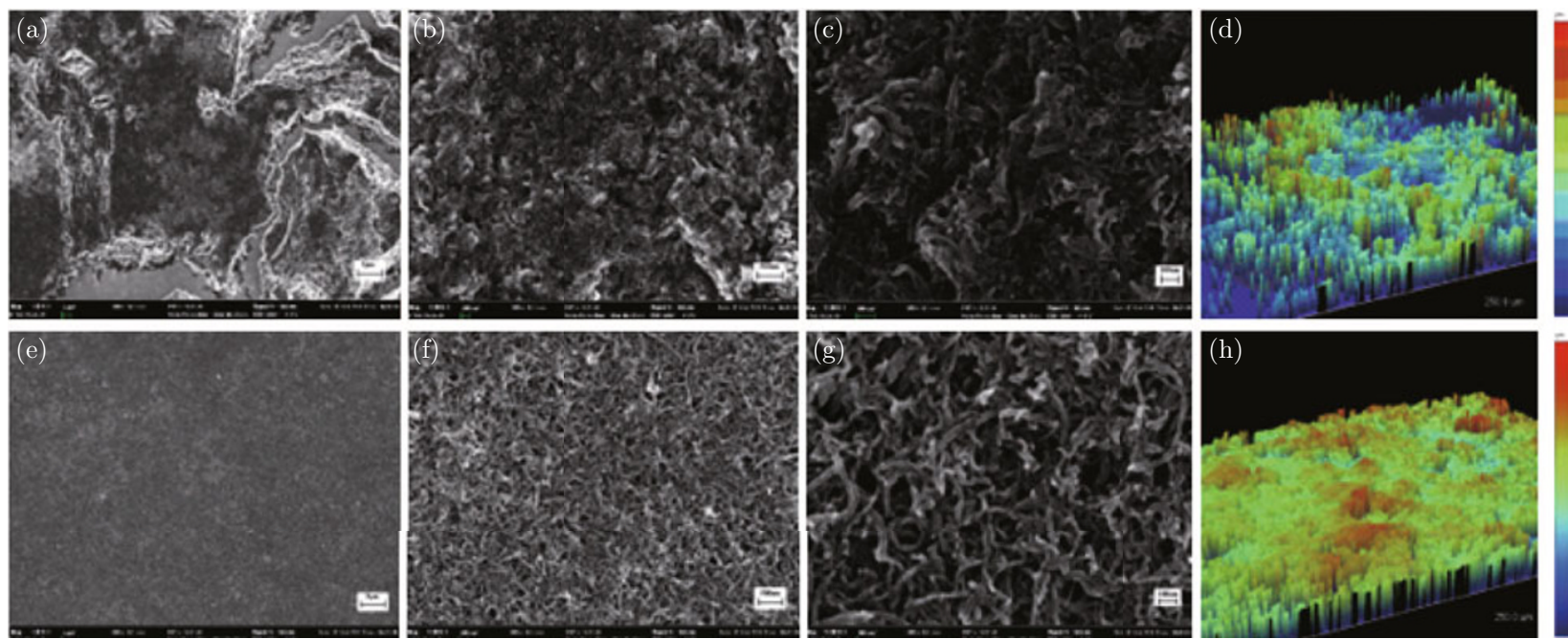

Fig. 5 SEM image and 3-dimensional image of EPD CNT layer with different amplified times. (a)(b)(c)(d), utilizing CNT before pretreatment; (e)(f)(g)(h), utilizing CNT after pretreatment. 


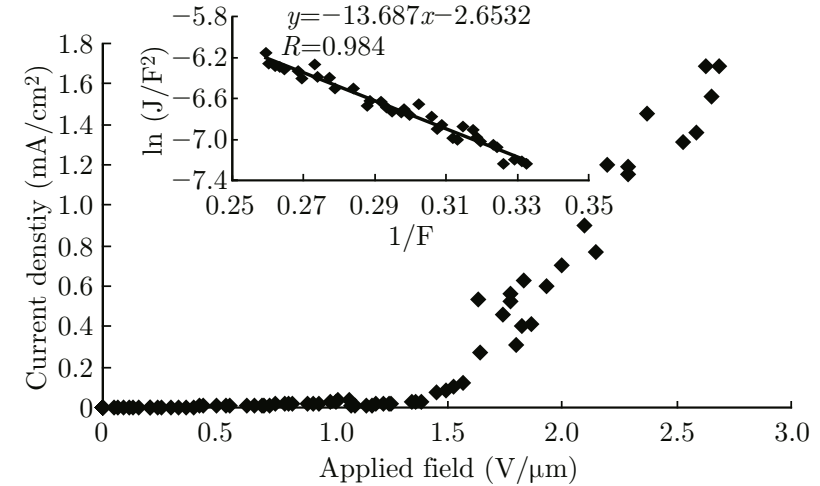

Fig. 6 Field-emission current density versus the applied electric field for the CNTs field emitters fabricated by EPD.

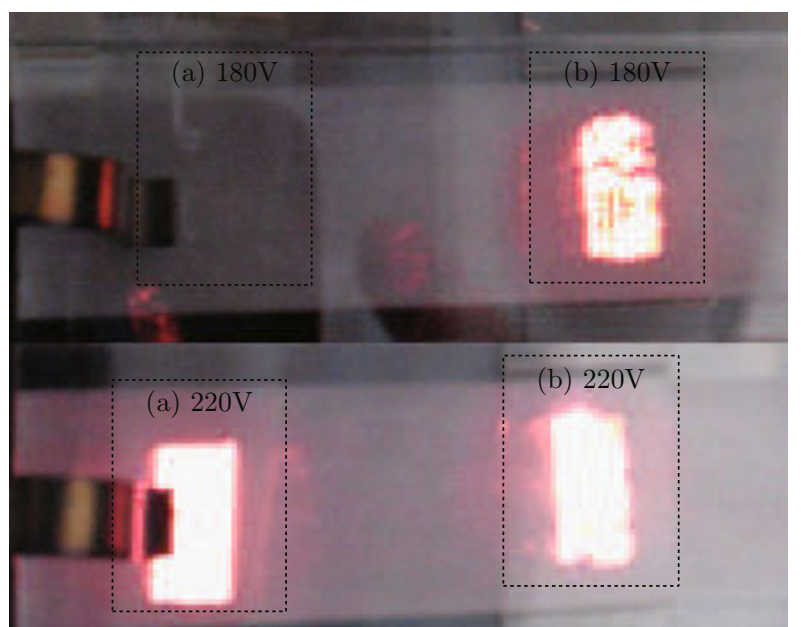

Fig. 7 Photograph of sustain discharge testing for PDP panel with (b) and without (a) EPD CNT layers.

\section{Conclusion}

The CNT layer has been demonstrated to reduce the high driving voltage in AC-PDP. The CNT layer, which was integrated on dielectric layer overlapping the PDP bus electrode, was formed by EPD without sheltering further plasma emission light. EPD layer with uniform and dense CNTs was deposited on given area of PDP panel. This morphology of CNT layer guarantees the excellent field-emission properties of low turn-on field. As a result, the minimum sustaining voltage was decreased by $30 \sim 40 \mathrm{~V}$ than that of the traditional ACPDP. Further work to investigate the effect to luminance efficiency using CNT materials for AC-PDP is in progress.

\section{Acknowledgments}

This work was supported by the National Natural Science Foundation of China (91023029) and the Shanghai Nano Projects (0952nm06300).

\section{References}

[1] In Cheol Song, Jung-Woo Ok, Seok Won Hwang, HoJun Lee, Chung-Hoo Park, Don-Kyu Lee and Hae June L, Thin Solid Films 518, 3122 (2010).

[2] Kwan Hyun Cho, Sung Il Ahn, Seong Min Lee and Kyung Cheol Choi, IEEE T. Electron Dev. 57, 2183 (2010). http://dx.doi.org/10.1109/TED . 2010. 2052693

[3] Chung-Hoo Park, Young-Kee Kim, Byeong-Eon Park, Woo-Geun Lee and Jung-Soo Cho, Mater. Sci. Eng. 60, 149 (1999). http://dx.doi.org/10.1016/ S0921-5107 (99) 00028-8

[4] G. S. Lee, J. Y. Lee, Y. B. Cheon, K. B. Kim, J. J. Kim and S. H. Sohn, Thin Solid Films 519, 3037 (2011). http://dx.doi.org/10.1016/j.tsf .2010.12.019

[5] Hae-Yoon Jung, Tae-Ho Lee, Ohyung Kwon, HeeWoon Cheong, Sven Ole Steinmüller, Jürgen Janek and Ki-Woong Whang, IEEE Elec. Dev. Lett. 31, 686 (2010). http://dx.doi.org/10.1109/LED. 2010. 2047236

[6] Seung-Hyeon Yang, Joeoong Hahn, Jaeik Han, JongDeok Lee and Tae-Seung Cho, IEEE T. Plasma Sci. 38, 1639 (2010). http://dx.doi.org/10.1109/TPS. 2010. 2047952

[7] Rakhwan Kim, Younghyun Kim and Jong-Wan Park, Vacuum 61, 37 (2001). http://dx.doi.org/10.1016/ S0042-207X (00) 00431-0

[8] Woo Hyun Kim, Kwan Hyun Cho and Kyung Cheol Choi, IEEE T Electron Dev. 57, 2644 (2010). http:// dx.doi .org/10.1109/TED . 2010. 2058850

[9] Sang Hoon Yoon, Jin-Seok Kim and Yong-Seog Kim, Curr. Appl. Phys. 6S1, e154 (2006).

[10] Akihiro Nakao, Yoshikazu Tanaka and Ari IdeEktessabi, Surf. Coat. Tech. 203, 2731 (2009). http:// dx. doi.org/10.1016/j. surf coat. 2009.02.102

[11] Van der Biest OO and L. J. Vandeperre, Annu. Rev. Mater. Sci. 29, 327 (1999). http://dx.doi.org/10. 1146/annurev.matsci.29.1.327

[12] A. R. Boccaccini and I. Zhitomirsky, Curr. Opin. Solid State Mater. Sci. 6, 251 (2002). http://dx.doi.org/ 10.1016/S1359-0286(02)00080-3

[13] B. J. C. Thomas, A. R. Boccaccini and M. S. P. Shaffer, J. Am. Ceram. Soc. 88, 980 (2005). http://dx. doi.org/10.1111/j.1551-2916.2005.00155.x

[14] B. J. C. Thomas, M. S. P. Shaffer, S. Freeman, M. Koopman, K. K. Chawla and A. R. Boccaccini, Proceedings of the 2nd International Conference on Electrophoretic Deposition: Key Engineering Materials 314, 141 (2006). http://dx.doi.org/10.4028/www. scientific.net/KEM.314.141

[15] J. M. Moon, K. H. An, Y. H. Lee, Y. S. Park, D. J. Bae and G. S. Park, J. Phys. Chem. B 105, 5677 (2001). http://dx.doi.org/10.1021/jp0102365

[16] J. Bae, Y. Yoon, S. Lee and H. Baik, Physica B 323, 169 (2002). http://dx.doi.org/10.1016/ S0921-4526 (02) 00890-6 
[17] D. Kurnosov, A. S. Bugaev, K. N. Nikolski, R. Tchesov and E. Sheshin, Appl. Surf. Sci. 215, 232 (2003). http://dx.doi.org/10.1016/ S0169-4332(03) 00307-6
[18] H. Ma, L. Zhang, J. Zhang, L. Zhang, N. Yao and B. Zang, Appl. Surf. Sci. 251, 258 (2005). http://dx. doi.org/10.1016/j.apsusc. 2005.03.100 